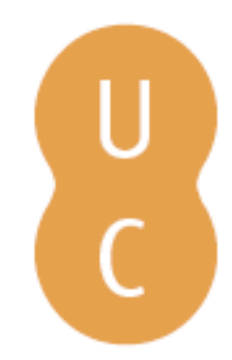

\title{
nommalina
}

\section{Desastres no Brasil: uma análise socio-espacial da vulnerabilidade institucional através da evolução da decretação municipal de situação de emergência e de estado de calamidade pública}

\author{
Autor(es): $\quad$ Valencio, Norma \\ Publicado por: Imprensa da Universidade de Coimbra; RISCOS - Associação \\ Portuguesa de Riscos, Prevenção e Segurança
}

URL

persistente:

DOI:

URI:http://hdl.handle.net/10316.2/34907

Accessed : $\quad$ 26-Apr-2023 15:07:30

A navegação consulta e descarregamento dos títulos inseridos nas Bibliotecas Digitais UC Digitalis, UC Pombalina e UC Impactum, pressupõem a aceitação plena e sem reservas dos Termos e Condições de Uso destas Bibliotecas Digitais, disponíveis em https://digitalis.uc.pt/pt-pt/termos.

Conforme exposto nos referidos Termos e Condições de Uso, o descarregamento de títulos de acesso restrito requer uma licença válida de autorização devendo o utilizador aceder ao(s) documento(s) a partir de um endereço de IP da instituição detentora da supramencionada licença.

Ao utilizador é apenas permitido o descarregamento para uso pessoal, pelo que o emprego do(s) título(s) descarregado(s) para outro fim, designadamente comercial, carece de autorização do respetivo autor ou editor da obra.

Na medida em que todas as obras da UC Digitalis se encontram protegidas pelo Código do Direito de Autor e Direitos Conexos e demais legislação aplicável, toda a cópia, parcial ou total, deste documento, nos casos em que é legalmente admitida, deverá conter ou fazer-se acompanhar por este aviso.

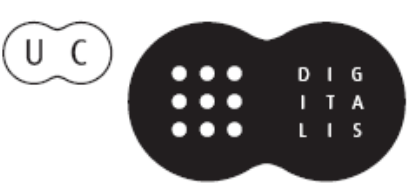



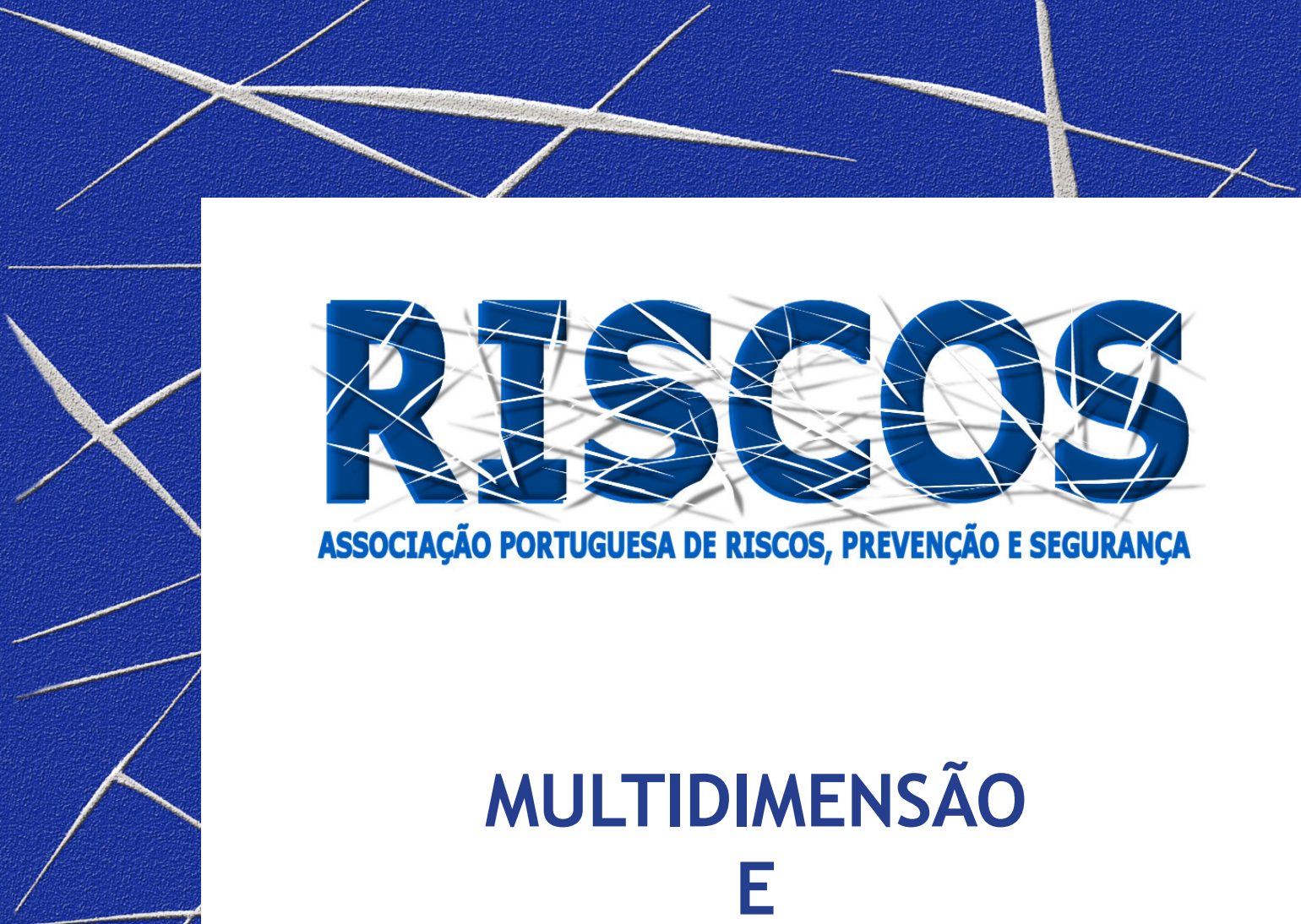

ASSOCIAÇÃO PORTUGUESA DE RISCOS, PREVENCCÃO E SEGURANÇA

MULTIDIMENSÃO

E
TERRITÓRIOS DE RISCO

III Congresso Internacional

I Simpósio Ibero-Americano

VIII Encontro Nacional de Riscos

Guimarães

2014 


\title{
DESASTRES NO BRASIL: UMA ANÁLISE SOCIO-ESPACIAL DA VULNERABILIDADE INSTITUCIONAL ATRAVÉS DA EVOLUÇÃO DA DECRETAÇÃO MUNICIPAL DE SITUAÇÃO DE EMERGÊNCIA E DE ESTADO DE CALAMIDADE PÚBLICA
}

\author{
Norma Valencio \\ Programa de Pós Graduação em Sociologia, Universidade Federal de São Carlos \\ Programa de Pós-Graduação em Ciências da Engenharia Ambiental, Universidade de São Paulo \\ normaf@terra.com.br
}

\begin{abstract}
RESUMO
O debate sociológico no tema dos riscos de desastres tem enfatizado os problemas decorrentes da lógica tecnocrática que permeia a gestão pública das emergências. A racionalidade burocrática e autoritária, própria do ethos tecnocrático, obstaculiza uma atuação técnico-operacional compatível com as demandas protetivas da sociedade civil. Disso resultam novos processos de esgarçamento do tecido socioambiental, dentre eles, a ampliação dos desastres. Esse estudo focaliza tal problemática no Brasil e a ilustra através da evolução do ato administrativo de decretação municipal de situação de emergência (SE) e de estado de calamidade pública (ECP), reconhecidos por portaria da Secretaria Nacional de Defesa Civil do Ministério da Integração Nacional (SEDEC/MI), no decênio 2004-2013. Foram identificados aspectos temporais e espaciais relevantes que subsidiam a discussão acerca da vulnerabilidade institucional do Sistema Nacional de Proteção e Defesa Civil (SINPDEC). Apoio: CNPq.

Palavras-chave: Sociologia dos Desastres; Defesa Civil; Brasil.
\end{abstract}

\section{Introdução}

No Brasil, a gestão pública das emergências e dos desastres apresenta um ethos tecnocrático, o qual orienta as práticas de seu quadro humano, predominantemente, para a atuação na resposta - isto é, para o resgate e reabilitação imediata dos grupos atendidos - ao invés da atuação de cunho preventivo ou recuperativo. Esse tipo de gestão tem acentuado o distanciamento entre os repertórios interpretativos da realidade concreta adotados pelo meio técnico-operacional de defesa civil e pelos grupos sociais afetados, o que resulta numa desconexão entre as expectativas e as práticas de ambos.

Convém refletir se, porventura, a acomodação do Sistema Nacional de Proteção e Defesa Civil (SINPDEC) na referida lógica estaria prestes a se revelar como uma armadilha auto-imposta; isto é, estaria explicitando o anacronismo da engrenagem institucional devido a crescente incapacidade de cumprir a missão em nome da qual foi criada.

\section{"Desastres não geram efeitos..."}

Muito a calhar, é a reflexão de Dombrowsky (1998) de que desastres não geram efeitos, mas são efeitos de um processo sociopolítico deteriorado. Os aspectos físicos da devastação não são autoexplicativos e o seu desvelamento depende do quanto se saiba acerca da crise social por detrás da ocorrência (Quarantelli, 2005; Alexander, 2005; Tierney, 2007). A resistência institucional em aceitar essa abordagem é imensa em sociedades onde vigora o patrimonialismo no seio do Estado, como no Brasil, porque os seus agentes se favorecem de práticas autocráticas. Tal como em outros contextos latino-americanos, como os mencionados por Lavell (1993), no Brasil o mainstream científico pactua com a gestão tecnocrática e difunde um discurso que inviabiliza perceber a complexidade social inerente aos desastres. Nesse discurso, focaliza-se a 
predição e o monitoramento strictu, com destaque aos eventos da natureza. A discussão pautase por parâmetros físicos e por tecnologias sofisticadas de difícil acesso social. 0 establishment científico tem se tornado massivamente institucionalizado e opressivamente hierárquico (Ingold, 2000), criando disputas científicas assimétricas e instrumentalizadas pelos interesses ocultos nas tramas do Estado e do mercado. 0 faz de modo a desqualificar tanto a perspectiva relacional exigida pela discussão dos temas complexos, como o dos desastres, quanto o repertório empírico de quem vivencia tais acontecimentos trágicos. Uma das estratégias de desqualificação é atribuir o qualificativo 'natural' a certos tipos de desastres - não por acaso, a maioria deles - a fim de esvaziar a possibilidade de identificação das responsabilidades públicas (Douglas e Wildawsky, 1982).

\section{Os desastres no contexto institucional brasileiro}

Se observarmos o contexto de ocorrência de desastres no Brasil, vemos que as autoridades nomeiam como 'naturais' a sua quase totalidade (mais de $90 \%$ ). O foco das explicações institucionais sobre os desastres havidos recai sobre as especificidades do evento - no geral, de origem hidrometeorológica - até concluir que se tratou de um perigo inusitado. É dessa explicação que se extrai a justificativa institucional para não se estar adequadamente preparado nem ter promovido a preparação comunitária devida, associando à ideia de que só restaria lidar com uma parcela das consequências (perdas e danos), no geral, movimentando a indústria do desastre (Valencio, 2014).

No decênio 2004-2013, foi preocupante tanto a evolução dos atos administrativos de decretação municipal de situação de emergência (SE) e de estado de calamidade pública (ECP) no país, reconhecidos pelo ente federal através de portaria emitida pela Secretaria Nacional de Defesa Civil do Ministério da Integração Nacional (SEDEC/MI), quanto a sua média anual. Durante a primeira gestão Lula (2003-2006), o percentual anual de decretações municipais de emergência (SE e ECP) em relação ao total dos municípios brasileiros foi de $21,25 \%$. Na segunda gestão Lula (2007-2010), foi de 22,75 \%. Já na gestão Dilma, em fase de finalização (recorte de janeiro de 2011 a agosto de 2014), em média, anualmente, 31,56\% dos municípios brasileiros declarou emergência. Em média, no país e a cada ano, 1/3 das decretações de emergência se repetem ou se estendem temporalmente no mesmo município. Isso indica que as autoridades locais não conseguiram equacionar o problema, ainda que com o auxílio dos níveis superiores de governo (estadual e federal). Esse é também um indício da debilidade estrutural do SINPDEC frente ao objetivo de tomar providências para uma recuperação mais duradoura do espaço onde os cidadãos vivem e convivem. No referido decênio, a média anual de 1.944 decretações de desastre (SE e ECP) por autoridades municipais, com o devido endosso federal, ocorre num universo de 5.565 municípios.

Ao distribuir os decretos de SE e ECP nas cinco (dentre 27) Unidades da Federação (UF) que apresentaram o maior número de emergências no período 2004-2013, identifica-se que as mesmas são integrantes das macrorregiões mais ricas e mais pobres do país, a saber, as macrorregiões Sul $(I D H=0,830)$ e Nordeste $(I D H=0,716)$. Tais UFs - a saber, Rio Grande do Sul, Santa Catarina, Ceará, Paraíba e Bahia - concentram 10.183 das ocorrências, o que representa $52,36 \%$ do total. O percentual de municípios, pertencentes a essas UFs, que vivenciou emergência mais de uma vez, no referido período, é superior a 70\%. A repetição de um mesmo tipo de desastre, nos mesmos moldes - isto é, na similaridade dos perigos e nas características das perdas e danos humanos, materiais e ambientais -, paira por sobre o Estado brasileiro como 
se não houvesse lição a extrair a cada ocorrência ou, ainda, como se os eventuais processos adaptativos em curso, que não resultam eficazes, não precisassem ser problematizados.

\section{Conclusões}

Para finalizar, enumeraremos três fios da teia tecnocrática que urge ser desmanchada, a saber:

(a) o processo de crescimento da estrutura institucional de defesa civil, que é calcado na ampliação de recursos humanos e tecnológicos voltados para o monitoramento de condições atmosféricas, hidrológicas, de estabilidade dos terrenos sujeitos aos escorregamentos de massa e afins, mas denomina isso, equivocadamente, como monitoramento de desastres. Falta uma parte expressiva de variáveis nessa equação;

(b) a cronicidade de decretação de emergências em um conjunto de municípios e UFs, o que gera uma banalização daquilo que Agamben (2004) define como Estado de exceção. Isto é, a máquina pública local (ou estadual) considera normal adotar um modus operandi não democrático para deliberar e gerir recursos orçamentários e extraordinários e estabelecer parcerias com a indústria do desastre. Isso enseja um ambiente institucional propício ao aumento da corrupção e de práticas correlatas, que são frequentes num contexto histórico e institucional patrimonialista. Enquanto isso, as comunidades afetadas recebem providências de reabilitação de baixa qualidade e são paulatinamente abandonadas à própria sorte (Valencio, 2012);

(c) a forma autocrática de condução do modelo econômico neodesenvolvimentista, o que impede a discussão pública sobre alternativas de desenvolvimento que ampliem a segurança humana no contexto dos desastres. O neodesenvolvimentismo das gestões Lula e Dilma é caracterizado por apelos nacionalistas, populistas e policlassistas, cujo programa essencial é o crescimento econômico que não rompa com os limites do modelo neoliberal das gestões FHC e é modelo sobre o qual se torna socialmente incorreto manifestar oposição (Boito Jr., 2012).

Nessa teia, é possível identificar aquilo que Hewitt (1995) chama de missing voices. Aqueles que não têm recursos de voz são invisíveis, ignorados ou marginalizados no mainstream da agenda pública (Douglas, 1992), o que ocasionará o que Hewitt (1995) denominará de missing agendas. O número significativo de decretos de emergência ao ano aponta para a existência de uma crise crônica no contexto nacional, razão pela qual é possível supor que a forma como a mesma tem sido interpretadas institucionalmente seja uma das causas de sua persistência. Indaga-se: os órgãos de defesa civil, articulados no SINPDEC, estariam, de fato, aptos a cumprir a sua missão institucional, se os números oficiais os desdizem? Até que ponto as comunidades desassistidas e fragilizadas aguentarão seus níveis crescente de vulnerabilidade sem questionar a lógica tecnocrática?

Pelos vícios que a teia acima mencionada suscita, consideramos que a repolitização do debate em torno dos rumos institucionais de defesa civil seria salutar e providencial. 0 avanço da decretação dos desastres, pelo Brasil adentro, esvazia paulatinamente as conquistas democráticas e flexibiliza os direitos dos grupos sociais afetados. Uma vez aceita a decretação, os agentes institucionais impõem o discurso sobre aquilo que será entendido como perigo e vulnerabilidade; promovem um novo ordenamento territorial (socialmente higenista); definem prioridades e o conteúdo do atendimento emergencial aos que estão em circunstância de vida nua (os espaços e regras de acolhimento, as opções de alimentação, as cotas de abastecimento 
hídrico e afins); gerem autonomamente os recursos públicos descartando o controle social, entre outros.

A lógica tecnocrática institucional em defesa civil se vale de uma confusão deliberada entre eventos, perigos e desastres. Assim, impede que o imaginário social compreenda, com maior clareza, que os eventos hidrometeorológicos severos ou extremos que, eventualmente, se manifestem não devam ser mecanicamente interpretados como sinônimos de perigos; se, porventura, as características intrínsecas e espaciais de manifestação de um desses eventos específicos o torna um perigo, do mesmo não decorre, necessariamente, um desastre. Quando as componentes sociais são adicionadas à explicação, o discernimento entre evento, perigo e desastre é facilitado. Contudo, essa adição implica questionar a prática política que baliza a soluções técnicas ora adotadas e, de resto, questionar os modos precários de garantir direitos territoriais, de morar, de circular e de trabalhar de milhões de pessoas que, de tal ou qual forma, integram famílias e comunidades indefesas no Brasil. Num contexto de milhões de semfala e sem-agenda, os desastres aumentam no Brasil. Isto é, aumentam em ordem direta aos sentimentos de desamparo e de desfiliação social os quais não derivam, primariamente, da experiência extrema de privação material e de desestruturação das rotinas da vida cotidiana, que um desastre suscita, mas de uma ordem pública que pode desumanizar a sociedade civil.

\section{Bibliografia}

AleXANDer, David E. (2005) - “An interpretation of disasters in terms of changes in culture, society and international relations”. In: Perry, Ronald W.; Quarantelli, Enrico L. (Eds.) - What is a disaster? New answers to old questions. Newark, International Research Committee on Disasters, p. 25-38.

Agamben, Giorgio (2004) - Homo sacer: o poder soberano e a vida nua. Belo Horizonte, Editora da UFMG, $197 p$.

Bolto JR., Armando (2012) - “As bases políticas do neodesenvolvimentismo”. Trabalho apresentado na edição de 2012 do Fórum Econômico da Fundação Getúlio Vargas, São Paulo. Disponível em: http://www. eesp.fgv.br/sites/eesp.fgv.br/files/file/Painel\%203\%20-\%20Novo\%20Desenv\%20BR\%20-\%20Boito\%20 -\%20Bases\%20Pol\%20Neodesenv\%20-\%20PAPER.pdf Acessado em: 14 de janeiro de 2013.

Dombrowsky, Wolf R. (1998) - “Again and again: is a disaster what we call a “disaster”?”. In: QuARANTElLI, EnRICO L. (org.). What is a disaster? Perspectives on the question. London/New York, Routledge, p. 19-30.

Douglas, Mary (1992) - Risk and blame: essays in cultural theory. London/New York, Routledge, 336 p.

DOUglas, Mary; WILDAVSKY, Aaron (1982) - Risk and culture - and essay on the selection of technological and environmental dangers. Berkeley, University of California Press, 232 p.

HEWITT, Kenneth (1995) - “Excluded perspectives in the social construction of disaster". International Journal of Mass Emergencies and Disasters, v. 13, n. 3, Mattoon, p. 317-339.

INGOLD, Tim (2000) - The perception of the environment: essays on livelihood, dwelling and skill. London and New York, Routledge, 2000, 462 p.

LaVelL, Allan (1993) - “Ciencias Sociales y Desastres Naturales en América Latina: un encuentro inconcluso”. In: Maskrey A. (org. ). Los desastres no son naturales. Panamá, Rede de Estudios Sociales en Prevención de Desastres en América Latina, p. 111-127.

QuARANTELLI, Enrico L. (2005) - “A social science research agenda for de disasters of the $21^{\text {st }}$ century: theoretical, methodological and empirical issues and their professional implementations". In Perry, Ronald W.; Quarantelli, EnRIco L. (Eds.) - What is a disaster? New answers to old questions. USA, International Research Committee on Disasters, p. 325-396. 


\section{CAPÍTULO 3.5: RISCOS SOCIAIS NO CONTEXTO DE CRISE GLOBAL}

TierneY, K. J. (2007) - “From the margins to the mainstream? Disaster research at the crossroads". Annual Review of Sociology. Vol. 33, p.p.503-525. DOI: 10.1146/annurev.soc.33.040406.131743

VALENCIO, Norma (2012) - Para além do desastre: O caso brasileiro. Curitiba, Editora Appris, 227p.

VAlencio, Norma F. L.S. (2014) - “Desastres, tecnicismo e sofrimento social”. Ciência \& Saúde Coletiva, Rio de Janeiro, v. 19, n. 9, p. 3631-3644. 